\title{
Spectral function of the electron in a superconducting resonating valence-band state
}

\author{
V. N. Muthukumar, ${ }^{1}$ Z. Y. Weng, ${ }^{2}$ and D. N. Sheng ${ }^{3}$ \\ ${ }^{1}$ Department of Physics, Princeton University, Princeton, New Jersey 08544 \\ ${ }^{2}$ Center for Advanced Study, Tsinghua University, Beijing 100084, People's Republic of China \\ ${ }^{3}$ Department of Physics and Astronomy, California State University, Northridge, California 91330
}

(Received 19 November 2001; published 6 June 2002)

\begin{abstract}
We present a model calculation of the spectral function of an electron in a superconducting resonating valence-bond (RVB) state. The RVB state, described by the phase-string mean-field theory of the $t$ - $J$ model, is characterized by three important features: (i) spin-charge separation, (ii) short-range antiferromagnetic correlations, and (iii) holon condensation. We show that when a bare hole is created, as in photoemission spectroscopy for instance, the RVB background responds with an antiferromagnetic oscillation of the local spin density surrounding the hole. This leads to matrix element effects with definite experimental consequences. We compare our results with data obtained from angle resolved photoemission spectroscopy in superconducting $\mathrm{Bi}_{2} \mathrm{Sr}_{2} \mathrm{CaCu}_{2} \mathrm{O}_{8+\delta}$ at optimal doping concentration.

DOI: 10.1103/PhysRevB.65.214522

PACS number(s): 74.20.Mn, 75.10.Jm, 74.72.- h, 71.27.+a
\end{abstract}

\section{INTRODUCTION}

The spectral function of an electron, $A(\mathbf{k}, \omega)$, being the probability of finding an electron with momentum $\mathbf{k}$ and energy $\omega$, is a fundamental quantity in any description of interacting electrons. In this paper, we consider the spectral function of an electron described by the $t-J$ model, a deceptively simple model describing the motion of holes in an antiferromagnetic background. The model Hamiltonian is thought to capture the underlying physics of the high-temperature superconductors (HTSC), and is the basic Hamiltonian for most theories of the resonating valence-band (RVB) state. In these theories, the competition between the superexchange and hopping processes leads to strong correlations between the electrons. Consequently, the electron is not the elementary excitation of the system and the low-energy properties are dominated by collective charge and spin degrees of freedom. When such a system is probed, say, by photoemission spectroscopy, the photohole decays into a spinless holon and a neutral spinon excitation. Consequently, the spectral function is intrinsically broad and does not necessarily have a well defined peak in the normal state. In the superconducting state, the holons are condensed, and a quasiparticle peak is expected. A direct experimental technique that can be used to investigate these properties is angle-resolved photoemission spectroscopy (ARPES). In the last decade, ARPES measurements have contributed much to our understanding of the HTSC. Measurements done in the normal and the superconducting state of the HTSC reveal a variety of interesting features. ${ }^{1}$ For instance, the normal-state spectral function is extremely broad, with the broad peak evolving into a hump at $T_{c}$. In addition to the hump, a very sharp peak appears below $T_{c}$ at low binding energies. This is observed most clearly near the Brillouin-zone boundary around the $M$ points, $(0, \pm \pi)$ and $( \pm \pi, 0){ }^{2}$ The strength of the sharp peak appearing below $T_{c}$ is proportional to the superfluid density. ${ }^{3,4}$ Along the direction $(0,0) \rightarrow(\pi, \pi)$, there is some debate if the data below $T_{c}$ can be interpreted as showing a clear break between a coherent quasiparticle part and the broad incoherent background. ${ }^{5,1}$ Some of these features, as well as the effect of charge stripes on the ARPES spectra have been studied by a quantum Monte Carlo simulation of the $t$ - $J$ model (and its variants). ${ }^{6}$

It was first noted by Anderson that spin-charge separation, as envisaged by theories of the RVB state, may provide a natural explanation for the ARPES results. ${ }^{7}$ However, as we shall demonstrate in this paper, the inclusion of antiferromagnetic correlations present in an RVB state is very important in determining the spectral function. This has been pointed out earlier, in the context of photoemission from a Mott insulator. ${ }^{8}$ In this paper, we consider the doped Mott insulator, as described by the $t$ - $J$ model. We restrict our attention to the superconducting state, and calculate the spectral function. The calculational scheme incorporates three important features: (i) short-range antiferromagnetic (AF) correlations, (ii) spin-charge separation, and (iii) holon condensation.

The description of the RVB state and holon condensation is based on the bosonic RVB theory developed earlier by one of us. ${ }^{9,10}$ In this paper, we show that this scheme can be used to calculate the spectral function of the quasiparticle when the short-range AF correlations are taken into account. We find that a photohole creates oscillations of the spin density in its vicinity. Since, in the RVB picture, the spectral function is obtained as a convolution of spinon and holon spectral functions, the short-range correlations cannot be neglected even if we are interested in low-energy spectral properties of the electron. Unfortunately, the very nature of these correlations involves short-distance physics and we do not, at present, have a controlled scheme to incorporate such effects perturbatively. Instead, we follow an empirical approach. We first provide numerical results to demonstrate the presence of such AF correlations when a photohole is created. We then show that these results can be incorporated into the RVB (mean-field) description of spinons by including a $k$-dependent prefactor in the equation of motion for the spinons. The spectral function of the electron is then calculated by convolving the holon and spinon spectral functions. We compare our calculations with experiment and show that our description provides a consistent explanation for many of 
the generic features observed in the superconducting state of optimally doped Bi 2212. We also show that the results are qualitatively different if the short-range AF correlations are neglected.

The paper is organized as follows. In Sec. II, we provide a brief description of the superconducting RVB state, determined by a mean-field theory of the $t-J$ model. We then show that the spin defect created by a bare hole in the RVB state is accompanied by oscillations of the local spin density surrounding the hole. We provide a scheme to incorporate such short-range antiferromagnetic correlations in determining the spectral function. In Sec. III, we present our results for the spectral function and compare them with data obtained from ARPES in superconducting Bi 2212. The paper also discusses the difference between the phase-string and slave-boson mean-field theories of the RVB state.

\section{A SUPERCONDUCTING RVB STATE}

\section{A. Mean-field theory}

We begin by outlining briefly, the phase-string mean-field theory of the $t-J$ model. We summarize the basic results for completeness and the reader is referred to earlier papers for details. ${ }^{9,10}$ The phase-string mean-field theory is based on the operator decomposition of the electron,

$$
c_{i \sigma}=h_{i}^{\dagger} b_{i \sigma} e^{i \hat{\Theta}_{i \sigma}},
$$

where $h_{i}^{\dagger}$ is the bosonic holon creation operator, and $b_{i \sigma}$ is a spinon operator that is also bosonic. The fermionic nature of the electron is reflected by the field $\hat{\Theta}_{i \sigma}$, which originates from the fact that a hole moving through an AF background picks up sequential \pm signs on scrambling the $\mathrm{AF}$ order. The phase factor is therefore called the "phase string," referring to the string of scrambled spins created by the motion of the hole. In the superconducting state, the holons are Bose condensed with $\left\langle h_{i}^{\dagger}\right\rangle=h_{0} \sim \sqrt{\delta}, \delta$ being the hole concentration. Then, the decomposition of the electron above can be rewritten as

$$
c_{i \sigma}=h_{0} a_{i \sigma}+c_{l \sigma}^{\prime},
$$

where $a_{i \sigma} \equiv b_{i \sigma} e^{i \hat{\Theta}_{i \sigma}}$, is the (fermionic) composite spinon operator, and $c_{i \sigma}^{\prime}=\left(: h_{i}^{\dagger}:\right) a_{i \sigma}$, with $: h_{i}^{\dagger}: \equiv h_{i}^{\dagger}-h_{0}$. Accordingly, the single-particle Green's function for the electron $G_{e}$ is given by

$$
G_{e} \approx h_{0}^{2} G_{a}+G_{e}^{\prime},
$$

where $G_{a}$ is the Green's function of the $a$ field, and $G_{e}^{\prime}$ is a convolution of the holon and spinon Green's functions. Clearly, the spectral function of the electron, $A_{-}^{e}(\mathbf{k}, \omega)$ can now be expressed in terms of the spectral function of the composite spinons, $\rho_{a}$ and that of the holons, $\rho_{h}$.

Let us first consider the holon degrees of freedom. In the phase-string mean-field theory, the effective Hamiltonian $H_{h}$ for the holons is obtained by rewriting the original $t$ - $J$ Hamiltonian using the operator decomposition above. One then finds

$$
H_{h}=-t_{h} \sum_{\langle i j\rangle} e^{i A_{i j}^{f} h_{i}^{\dagger} h_{j}+\text { h.c. }, ~}
$$

where $A_{i j}^{f}$ represents the gauge field due to the spinons seen by the holons. At $T=0$, all spinons are paired and the meanfield theory leads to the result $\Sigma_{\square} A_{i j}^{f} \approx-\pi$, where the summation is over an elementary plaquette. ${ }^{9}$ Therefore, the superconducting state is described by paired spinons and a Bose condensate of holons that experience a $\pi$ flux around an elementary plaquette. The spectral function of the holons is then given by

$$
\rho_{h}(\mathbf{k}, \omega)=\cos ^{2} \frac{\theta_{\mathbf{k}}}{2} \delta\left(\omega-\epsilon_{\mathbf{k}-}^{h}\right)+\sin ^{2} \frac{\theta_{\mathbf{k}}}{2} \delta\left(\omega-\epsilon_{\mathbf{k}+}^{h}\right),
$$

where $\cos \theta_{\mathbf{k}}=\gamma_{\mathbf{k}} /\left(\sqrt{2} \lambda_{\mathbf{k}}\right)$. Here, $\gamma_{\mathbf{k}}=\cos k_{x} a+\cos k_{y} a, \lambda_{\mathbf{k}}$ $=\sqrt{\cos ^{2} k_{x} a+\cos ^{2} k_{y} a}$, and $\epsilon_{\mathbf{k} \pm}^{h}= \pm 2 t_{h} \lambda_{\mathbf{k}}-\mu_{h}$. At $T=0$, the holon chemical potential, $\mu_{h}=-2 t_{h} \lambda_{\mathbf{k}=0}=-2 \sqrt{2} t_{h}$.

The next step is to determine the spectral function of the composite spinon field $\rho_{a}$. To obtain $\rho_{a}$, we consider the equations of motion of the electron governed by the $t-J$ Hamiltonian rewritten in terms of the $h$ and $a$ fields. Neglecting multispinon processes, and invoking holon condensation as described in the previous paragraph, a closed set of equations can be obtained for the dynamics of the $a$ operators. ${ }^{10}$ This system of equations can be solved by introducing a Bogoliubov transformation

$$
a_{k \sigma} \propto u_{k} \gamma_{k \sigma}-\sigma v_{k} \gamma_{-k-\sigma}^{\dagger} .
$$

The linearized equation of motion ${ }^{10}$ can always be multiplied by an arbitrary $k$-dependent prefactor on the right hand side (rhs) of Eq. (6). It can only be fixed by going beyond this approximation, as will be discussed shortly. The operator $\gamma_{k}^{\dagger}$ creates an eigen excitation with energy

$$
E_{k}^{s}=\sqrt{\left(\epsilon_{k}-\mu\right)^{2}+\left|\Delta_{k}\right|^{2}},
$$

where $\Delta_{k}$ can be obtained self-consistently. ${ }^{10}$ For the purposes of this paper, however, we shall treat $\Delta_{k}$ as a parameter having $d$-wave symmetry, viz., $\Delta_{k}=\Delta_{0}\left(\cos k_{x} a-\cos k_{y} a\right)$. The quantities $u_{k}$ and $v_{k}$ are defined in the usual manner, as $u_{k}^{2}=\left[1+\left(\epsilon_{k}-\mu\right) / E_{k}^{s}\right]$, and $v_{k}^{2}=\left[1-\left(\epsilon_{k}-\mu\right) / E_{k}^{s}\right]$. The spectral function, $\rho_{a}$ is then given by

$$
\rho_{a}(k, \omega) \propto u_{k}^{2} \delta\left(\omega-E_{k}^{s}\right)+v_{k}^{2} \delta\left(\omega+E_{k}^{s}\right) .
$$

Using Eqs. (5), (7), and (3), the spectral function of the electron $A_{-}^{e}(\mathbf{k}, \omega)$ can be obtained. The results would then be qualitatively similar to the slave-boson $d$-wave mean-field theories of the RVB state. ${ }^{11}$ However, this analysis misses an important effect arising from the short-range AF correlations present in the RVB state. Consider the process of creating a bare hole (as in ARPES, for example) at a site $i$. From the operator decomposition (1), we see that the RVB state now has a holon at site $i\left(h_{i}^{\dagger}\right)$ where a spinon is destroyed $\left(a_{i \sigma}\right)$. The annihilation of a spinon in an RVB environment leads to a spin defect, whose presence causes AF oscillations of the local spin density around the site $i$. As we shall argue later in the paper, this effect has to be incorporated in the description of the spin degrees of freedom in order to get the correct 
form for the spectral function of the photohole. We will show that the effect of the AF oscillations is characterized (to leading order) by a $k$-dependent prefactor in the rhs of Eq. (7). The presence of such a prefactor leads to qualitative changes in the spectral function.

\section{B. Response to the creation of a bare hole in an RVB environment}

Consider a bare hole localized at site $i$, created by the action of the electron operator, $c_{i \sigma}$, on the ground state $\left|\Psi_{G}\right\rangle$. As we are primarily interested in determining the spin configuration around the hole, we focus on the quantity

$$
\left\langle\Psi_{G}\left|c_{i \uparrow}^{\dagger} S_{j}^{z} c_{i \uparrow}\right| \Psi_{G}\right\rangle .
$$

We determine this quantity within the same mean-field approximation invoked to solve the $t-J$ model. Using the decomposition (1), we get

$$
\begin{aligned}
& \left\langle\Psi_{G}\left|c_{i \uparrow}^{\dagger} S_{j}^{z} c_{i \uparrow}\right| \Psi_{G}\right\rangle \\
& =\left\langle\Psi_{G}\left|b_{i \uparrow}^{\dagger} \frac{1}{2} \sum_{\alpha} \alpha b_{j \alpha}^{\dagger} b_{j \alpha} b_{i \uparrow}\right| \Psi_{G}\right\rangle \\
& =-\frac{1}{2}\left|\left\langle\Psi_{G}\left|b_{i \uparrow}^{\dagger} b_{j \downarrow}^{\dagger}\right| \Psi_{G}\right\rangle\right|^{2}+\left.\frac{1}{2}\left\langle\Psi_{G}\left|b_{i \uparrow}^{\dagger} b_{j \uparrow}\right| \Psi_{G}\right\rangle\right|^{2} .
\end{aligned}
$$

It is instructive to compare Eq. (9) with results from the slave-boson description of the RVB state. As discussed earlier, the results obtained by a mean-field theory of the $a$ fields is akin to the slave-boson approach. Expressing Eq. (8) in terms of the $a$ operators, we get

$$
\begin{aligned}
& \left\langle\Phi_{G}\left|c_{i \uparrow}^{\dagger} S_{j}^{z} c_{i \uparrow}\right| \Phi_{G}\right\rangle \\
& \quad=-\frac{1}{2}\left[\left|\left\langle\Phi_{G}\left|a_{i \uparrow}^{\dagger} a_{j \downarrow}^{\dagger}\right| \Phi_{G}\right\rangle\right|^{2}+\frac{1}{2}\left|\left\langle\Phi_{G}\left|a_{i \uparrow}^{\dagger} a_{j \uparrow}\right| \Phi_{G}\right\rangle\right|^{2}\right] .
\end{aligned}
$$

Owing to the fermionic anticommutation relations of $a_{i \sigma}$, both terms on the rhs of Eq. (10) are negative. Note that $\left|\Phi_{G}\right\rangle$ in Eq. (10) denotes the corresponding mean-field ground state, different from $\left|\Psi_{G}\right\rangle$. In contrast, the phasestring mean-field theory leads to Eq. (9), where the two terms in the second line have opposite signs.

In the decomposition of Eq. (1), the spin sector of the problem is described by the Hamiltonian ${ }^{9}$

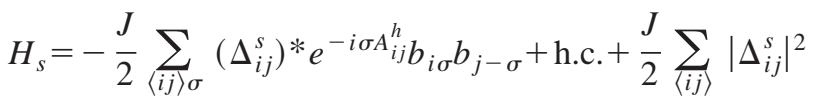

$$
\begin{aligned}
& +\lambda\left(\sum_{i \sigma} b_{i \sigma}^{\dagger} b_{i \sigma}-(1-\delta) N\right),
\end{aligned}
$$

where

$$
\left.\Delta_{i j}^{s}=\sum_{\sigma}<e^{-i \sigma A_{i j}^{h}} b_{i \sigma} b_{j-\sigma}\right\rangle
$$

is the RVB order parameter that is determined selfconsistently. The quantity $A_{i j}^{h}$ denotes the fictitious $\pi$-flux tubes bound to the holons seen by the spinons, and $\lambda$ is a Lagrange multiplier introduced to enforce the total spinon number. Recall that $\delta$ is the hole concentration. It should be noted that when $\delta \rightarrow 0$ (i.e., half filling), $A_{i j}^{h} \equiv 0$, and the RVB order parameter reduces to that of the Schwinger boson theory, which is known to account well for the shortrange $\mathrm{AF}$ correlations. ${ }^{12}$ The Hamiltonian $H_{J}$ can be diagonalized by a generalized Bogoliubov transformation. The details are found in Ref. 9. Using this mean-field solution, we obtain

$$
\left\langle\Psi_{G}\left|b_{i \uparrow}^{\dagger} b_{j \downarrow}^{\dagger}\right| \Psi_{G}\right\rangle=\left[(-1)^{i-j}-1\right] \sum_{m} u_{m} v_{m} w_{m \uparrow}^{*}(i) w_{m \uparrow}(j)
$$

and

$$
\left\langle\Psi_{G}\left|b_{i \uparrow}^{\dagger} b_{j \uparrow}\right| \Psi_{G}\right\rangle=\left[(-1)^{i-j}+1\right] \sum_{m} v_{m}^{2} w_{m \uparrow}^{*}(i) w_{m \uparrow}(j) .
$$

In the above, $u_{m}$ and $v_{m}$ are the "coherence factors" of the mean-field theory determined self-consistently for a given hole concentration $\delta$, within a Bogoliubov-de Gennes scheme, and $w_{m \sigma}$, a one-particle wave function. Equations (11) and (12) are now used in Eq. (9) to determine the spin density around the hole site $i$. The results are shown in Fig. 1, where we have plotted the spin density as a function of the hole distance along the $\hat{x}$ axis for hole concentrations $\delta=0$, and $\delta=1 / 7 \simeq 0.14$. As seen in the figure, the hole is surrounded by AF oscillations of the local spin density.

The oscillations shown in Fig. 1 is to be expected from Eq. (9) and we draw the reader's attention to the following. First, the oscillations in the local spin density are indicative of local AF correlations in the ground state $\left|\Psi_{G}\right\rangle$. Second, our result implies that the bare hole decays into elementary holon and spinon excitations that reside on different sublattices. Suppose the ground state $\left|\Psi_{G}\right\rangle$ is a singlet. Then, $c_{i \uparrow}\left|\Psi_{G}\right\rangle$ creates a holon and a spinon excitation with $S^{z}=-1 / 2$. Assuming the holon to be created on an odd sublattice site, our result suggests that the accompanying $S^{z}=-1 / 2$ spinon must be present primarily on the even sublattice sites as seen in Fig. 1. We see in the figure that the induced spins on the even sublattice sites point downwards. Besides the $S^{z}=-1 / 2$ spinon, multispinon excitations are also present in the bare hole state as an induced spin polaron, which is composed of nonvanishing up-spin density on the odd sublattice sites and an additional down-spin response on the even sites (besides the $S^{z}=-1 / 2$ spinon in Fig. 1). The effect of the multispinon excitations is beyond the scope of this paper and, as an approximation, will be neglected. 


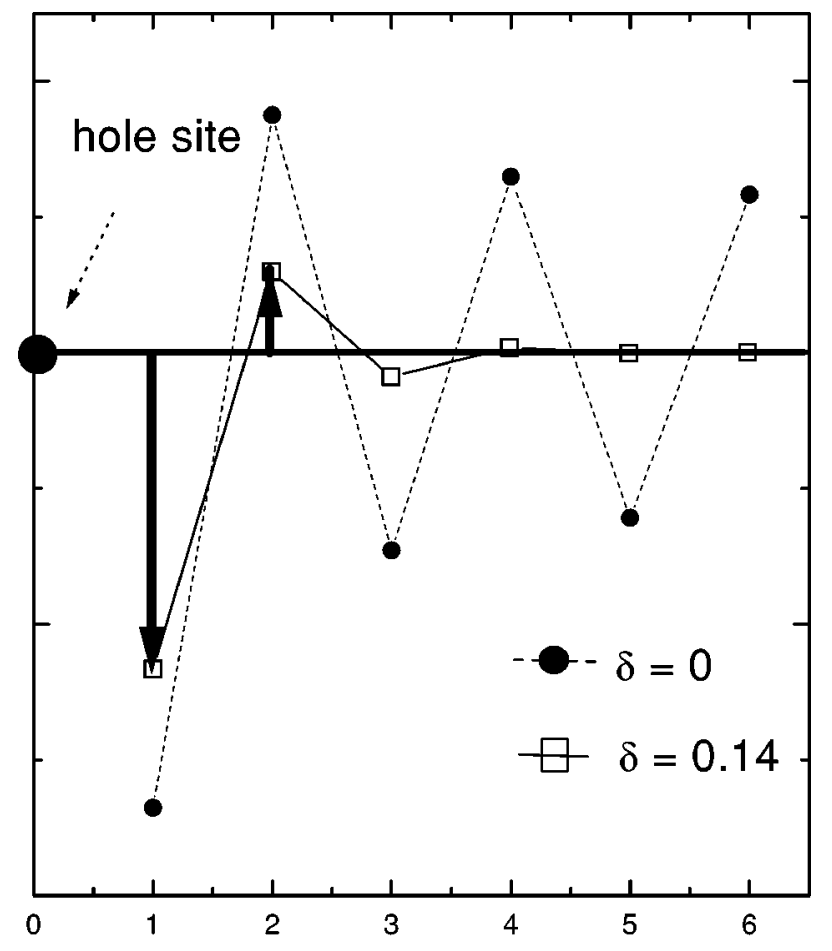

Distance from the hole along $x$-axis

FIG. 1. Spin configuration near an up-spin hole, created by $c_{\uparrow}$ on the ground state at $\delta=0$ and 0.14 , along the $\hat{x}$ axis.

Now let us consider the results obtained from a meanfield theory of the $a$ fields (or equivalently, the slave-boson mean-field theory). The AF oscillation shown in Fig. 1 is absent in this case. As is evident from Eq. (10), the $S^{z}=-1 / 2$ spinon is distributed around the holon smoothly without any density modulation. Hence, any description (such as the slave-boson theory) based on the dynamics of the $a$ fields misses this effect, at least, at the level of mean-field theory. At this point, it may be asked why any change in the local spin density should matter in the description of the spectral function at low energies. The answer is that the spectral function of the physical hole is obtained as a convolution of the spinon and holon fields and consequently, disturbances at short length scales do affect the low-energy dynamics of the hole. Therefore, we need a scheme to incorporate the effects that arise due to the composite nature of the $a$ field. In the following, we propose a scheme that can be incorporated at the level of mean-field theory and show that it leads to qualitative changes.

To build the AF correlations within the description of the $a$ fields, or equivalently, the modulation of the local spin density surrounding the hole site, we adopt the following approach. We write

$$
a_{i \uparrow}\left|\Psi_{G}\right\rangle \simeq \sum_{j} \eta_{j}(i) \alpha_{j \downarrow}^{\dagger}\left|\Psi_{G}\right\rangle+\text { multispinon terms }
$$

where $\eta_{j}(i) \neq 0$ only for $i$ and $j$ not belonging to the same sublattice, and $\alpha_{\mathbf{k} \sigma} \equiv\left|v_{\mathbf{k}}\right| \gamma_{\mathbf{k} \sigma}$ (the $d$-wave sign in $v_{\mathbf{k}}$ will be absorbed into $\eta$ ). We only retain the nearest- and thirdnearest-neighboring sites in the expansion (13); viz., $\eta_{j}(i)$ $=+(-) \eta_{0} / 2$, if $j=i \pm \hat{x}(\hat{y}) ; \quad \eta_{j}(i)=+(-) \eta_{1} / 2$, if $j=i$ $\pm 2 \hat{x}(\hat{x}) \pm \hat{y}(2 \hat{y}) ; \quad \eta_{j}(i) \approx 0$ for all other sites. The sign of $\eta_{j}(i)$ is from the $d$-wave symmetry of spinon pairing. Transforming to momentum space, we get, within this approximation, $a_{\mathbf{k} \uparrow}\left|\Psi_{G}\right\rangle \approx \eta_{\mathbf{k}}\left|v_{\mathbf{k}}\right| \gamma_{-\mathbf{k} \downarrow}^{\dagger}\left|\Psi_{G}\right\rangle$, where

$$
\begin{aligned}
\eta_{\mathbf{k}}= & \eta_{0}\left(\cos k_{x} a-\cos k_{y} a\right) \\
& +2 \eta_{1}\left(\cos k_{x} a \cos 2 k_{y} a-\cos k_{y} a \cos 2 k_{x} a\right) .
\end{aligned}
$$

Here we take $\eta_{1} / \eta_{0} \simeq 0.3$ for $\delta=0.14$, the choice suggested by the results in Fig. 1.

With this choice of the $k$-dependent prefactor, the equations of motion for the $a$ spinon are still solved. Recall that Eq. (6) was only written down up to an arbitrary $k$-dependent factor. Our choice of $\eta_{\mathbf{k}}$ fixes this factor. We reemphasize that this choice is not arbitrary and follows from the results shown in Fig. 1. The prefactor $\eta_{\mathbf{k}}$ is because the photohole is composed of the elementary holon and spinon excitations residing on different sublattices, reflecting the underlying AF correlations of the system. This structure is absent in the usual slave-boson mean-field theories.

One may also ask what happens when an electron is created (as, for example, in inverse photoemission), i.e., $c_{i \uparrow}^{\dagger}\left|\Psi_{G}\right\rangle$. It can be shown in this case that the $\uparrow$ spinon is created at the site $i$ with some residual amplitude extended over other sites of the same sublattice. Neglecting the residual amplitude, we get

$$
a_{\mathbf{k} \uparrow}^{\dagger}\left|\Psi_{G}\right\rangle \simeq u_{\mathbf{k}} \gamma_{\mathbf{k} \uparrow}^{\dagger}\left|\Psi_{G}\right\rangle
$$

which is essentially the same as the slave-boson result. In the above, $u_{k}^{2}=1-v_{k}^{2}$. While it is true that the two approaches still show some differences due to the presence (or absence) of short-range AF correlations, to leading order, the spinon density is peaked at site $i$ in both descriptions. Thus, the differences between the usual $d$-wave slave-boson mean-field theory and this calculation are seen mainly in the hole channel, owing to the momentum dependent prefactor $\eta_{\mathbf{k}}$. Hence, in the rest of the paper, we only focus on the hole channel (corresponding to the creation of a photohole in ARPES).

We now construct the spectral function of the electron using Eq. (3). It is easy to see that

$$
\begin{aligned}
& A_{-}^{\mathrm{e}}(\mathbf{k}, \omega) \\
& \quad=\theta(-\omega) \frac{1}{N} \sum_{\mathbf{k}} \int_{\omega}^{0} d \omega^{\prime} \rho_{h}\left(\mathbf{k}^{\prime}-\mathbf{k}, \omega^{\prime}-\omega\right) \rho_{a}\left(\mathbf{k}^{\prime}, \omega\right)
\end{aligned}
$$

where $\rho_{a}$ and $\rho_{h}$ are the spectral functions of the spinon and holon respectively. For obvious reasons, we write the spectral function as the sum of an incoherent and a coherent part, 
$A_{-}^{\mathrm{e}}=A_{-}^{\mathrm{i}}+A_{-}^{\mathrm{c}}$. Clearly, these two terms correspond to the two terms in the electron decomposition (3).

The coherent part of the spectral function is obtained from the contribution of the holon condensate. We get

$$
A_{-}^{\mathrm{c}}(\mathbf{k}, \omega)=\rho_{h}^{c} \eta_{\mathbf{k}}^{2} v_{\mathbf{k}}^{2} \delta\left(\omega+E_{\mathbf{k}}^{s}\right),
$$

where $\rho_{h}^{c} \propto \delta$ denotes the density of the holon condensate. This contribution is dubbed "coherent," since it is a sharp peak appearing below $T_{c}$ (the temperature at which the holons condense). The incoherent part of the spectral function is the convolution

$$
\begin{aligned}
A_{-}^{i}(\mathbf{k}, \omega)= & \frac{1}{N} \sum_{\mathbf{k} \prime}^{\prime} \eta_{\mathbf{k}^{\prime}+\mathbf{k}^{\prime}}^{2} v_{\mathbf{k}^{\prime}+\mathbf{k}}^{2}\left[\cos ^{2} \frac{\theta_{\mathbf{k}}}{2} \delta\left(\omega+E_{\mathbf{k}^{\prime}+\mathbf{k}}^{s}+\epsilon_{\mathbf{k}^{\prime}-}^{h}\right)\right. \\
& \left.+\sin ^{2} \frac{\theta_{\mathbf{k}}}{2} \delta\left(\omega+E_{\mathbf{k}^{\prime}+\mathbf{k}}^{s}+\epsilon_{\mathbf{k}^{\prime}+}^{h}\right)\right]
\end{aligned}
$$

The prime in the summation indicates that the contribution from the holon condensate is removed.

\section{RESULTS}

We are now in a position to plot $A_{-}^{\mathrm{e}}(\mathbf{k}, \omega)$ for various momenta and compare with results from ARPES. For the calculation of the spectral function, we choose the following parameters. The dispersion of the spinons $\xi_{\mathbf{k}}$ is determined by the nearest- and next-nearest-neighbor hopping integrals, $t_{1}=75 \mathrm{meV}, t_{2}=20 \mathrm{meV}$. The chemical potential, $\mu=-62$ $\mathrm{meV}$ is chosen to mimic the topography of the observed Fermi surface. We choose a value of $\Delta=20 \mathrm{meV}$ for the gap, and $t_{h}=4 t_{1}=0.3 \mathrm{eV}$ for the holon dispersion. Before proceeding to discuss the results for $A_{-}^{\mathrm{e}}(\mathbf{k}, \omega)$, we note that the following can be anticipated: (a) The coherent part of the spectral function, $A_{-}^{\mathrm{c}}(\mathbf{k}, \omega) \propto h_{0}^{2} \propto \delta$. So, the strength of the coherent peak will be proportional to the superfluid density, as observed experimentally. ${ }^{4,3}$ (b) As is evident from our results, $A_{-}^{\mathrm{c}}(\mathbf{k}, \omega)$ is strongly momentum dependent, owing to the factor $\eta_{\mathbf{k}}^{2}$. This contribution is strongest around the $M$ point and weakest around the $\Gamma$ point. (c) The incoherent part of the spectral function is expected to produce a broad background. Such a broad background is always seen in photoemission. Since, in our calculation, the background arises from a convolution of spinon and holon spectral functions, we expect a rather weak momentum dependence of the broad background. This may have already been observed experimentally. ${ }^{13}$

In Fig. 2, we show the results for $A_{-}^{\mathrm{e}}(\mathbf{k}, \omega)$ at a point on the Fermi surface, $\mathbf{k}_{f}=(0.4, \pi)$. We see clearly that the total spectral response is the sum of the incoherent and coherent pieces of the spectral function. The sharp peak at lower binding energy corresponds to $A^{\mathrm{c}}$ and the peak is located at an energy $\omega=E_{\mathbf{k}}^{s}$. The broad feature seen in the figure is the contribution from $A^{\mathrm{i}}$. The incoherent part exhibits a lowenergy edge around $E_{\mathbf{k}}^{s}$, and a broad peak ("hump") slightly above it. The origin of these features is very much the same as in the spectra of the undoped insulator. ${ }^{8}$ In both cases, the low-energy edge is determined by the dispersion of the spinon, $E_{\mathbf{k}}^{s}$. The origin of the hump lies in the local AF correlations embodied in the factor $\eta_{\mathbf{k}}$. Let us consider Eq. (17). The factor $\eta_{\mathbf{k}}$ is maximum at the $M$ point. As $\omega$ increases, $\mathbf{k}^{\prime} \neq 0$ terms in Eq. (17) have to contribute to the sum. However, for such terms, the factor $\eta_{\mathbf{k}+\mathbf{k}^{\prime}}$ decreases, thereby causing a hump in the spectral funtion. The position of the hump is shifted from the edge by $\epsilon_{\mathbf{k}^{\prime}-}^{h}$. A similar effect occurs in the photoemission of a single hole in the Mott insulator, where a broad hump arises from the coherence factors of the Schwinger boson mean-field theory. We emphasize that the factor $\eta_{\mathbf{k}}$ in the present case and the coherence factors in the Schwinger boson theory of the insulator reflect the antiferromagnetic correlations that play an important role in the formation of the observed humps. Seen in this perspective, the hump is not directly related to the 41-meV resonance observed in the neutron scattering, ${ }^{14}$ as conjectured in the literature ${ }^{15}$ though AF correlations clearly play a crucial role in both explanations.

In Fig. 3, we show the behavior of $A_{-}^{\mathrm{e}}(\mathbf{k}, \omega)$ for various $\mathbf{k}$ points. In the left panel, we show how the spectral function evolves as one moves away from the $M$ point in two perpen-

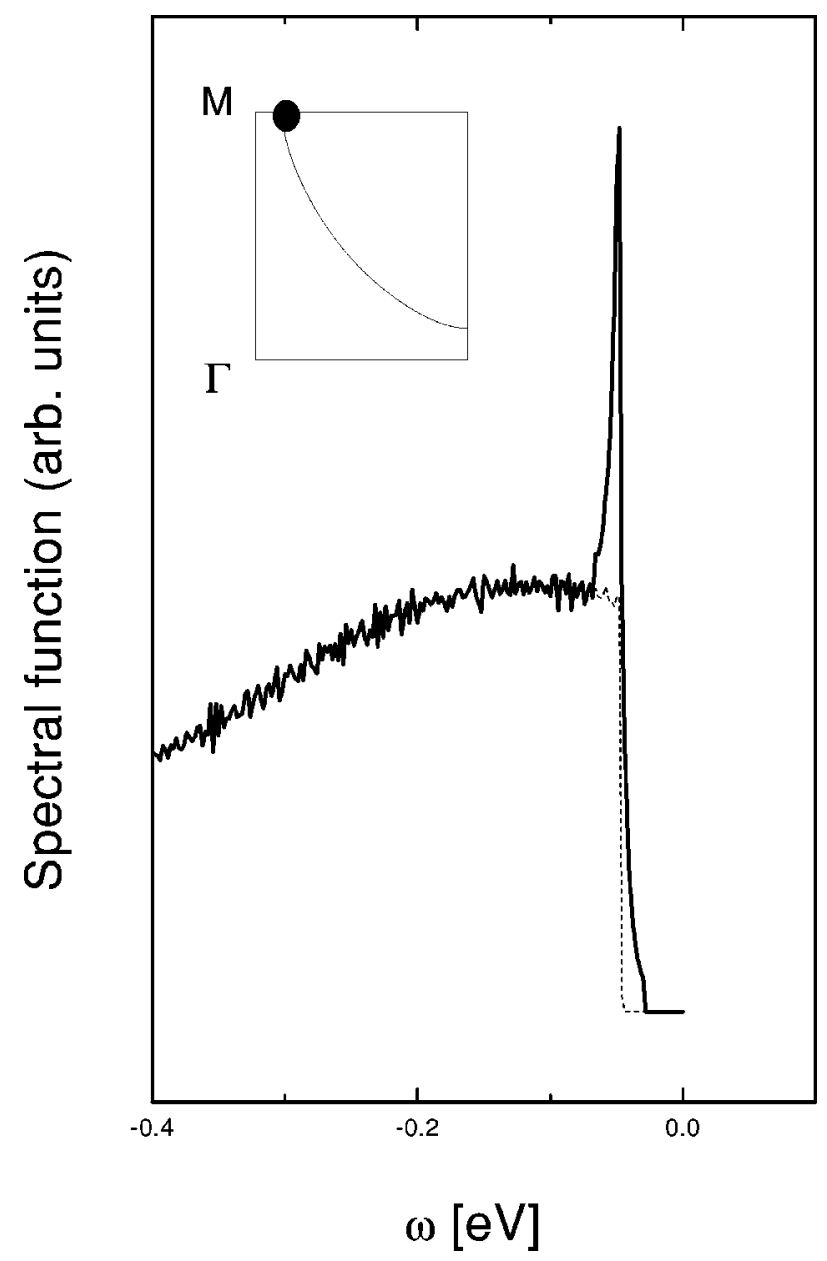

FIG. 2. The spectral function at $\mathbf{k}_{f}=(0.4, \pi)$, which is a Fermi point at the Brillouin-zone boundary (see the inset). The dashed curve shows only the incoherent part whose low-energy edge coincides with the coherent peak position while its 'hump' lies slightly above the edge. 

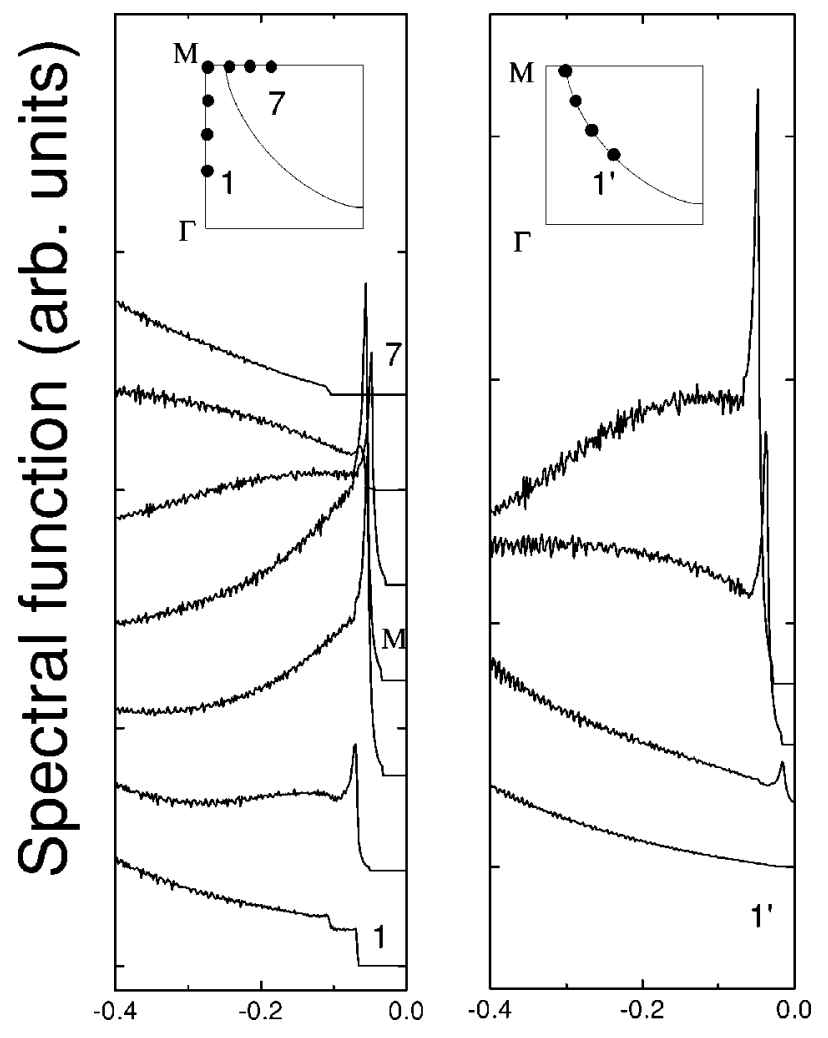

\section{$\omega[\mathrm{eV}]$}

FIG. 3. The spectral function at different momenta marked by full circles in the insets of two panels. The left panel corresponds to scans near the $M$ point along two directions, while the right represents momenta on the Fermi surface.

dicular directions. As can be seen in the figure, the peak-diphump structure gets more pronounced near the $M$ point. In the right panel, we show the spectral function for a series of points on the Fermi surface. Again, we see that the factor $\eta_{\mathbf{k}}$ causes the coherent peak as well as the hump to diminish away from the $M$ point. In particular, along the direction $\Gamma \rightarrow(\pi, \pi)$, these features are absent in our calculation. Though this is an approximate result, it shows that the inclusion of AF correlations suppresses the peak-dip-hump structure along this direction. Experimentally, there is some debate about the existence of a sharp quasiparticle peak along this direction. One group ${ }^{1}$ claims that the data below $T_{c}$ show a clear break between the broad background and a feature that emerges at low temperatures. Another group 5 holds that the line shape of the spectra along this direction evolve continuously as the temperature is lowered. In either case, the authors agree that the spectra are very different from those near the $M$ point. ${ }^{15}$ Within our approach, this is due to the AF oscillations created by the presence of the hole. As discussed in Sec. II, these oscillations lead to a matrix element effect dominated by terms with $d$-wave symmetry, (14). Therefore, the spectra near the $M$ point are bound to be different from the spectra along the nodal direction. In this context, we note that our results for the spectra

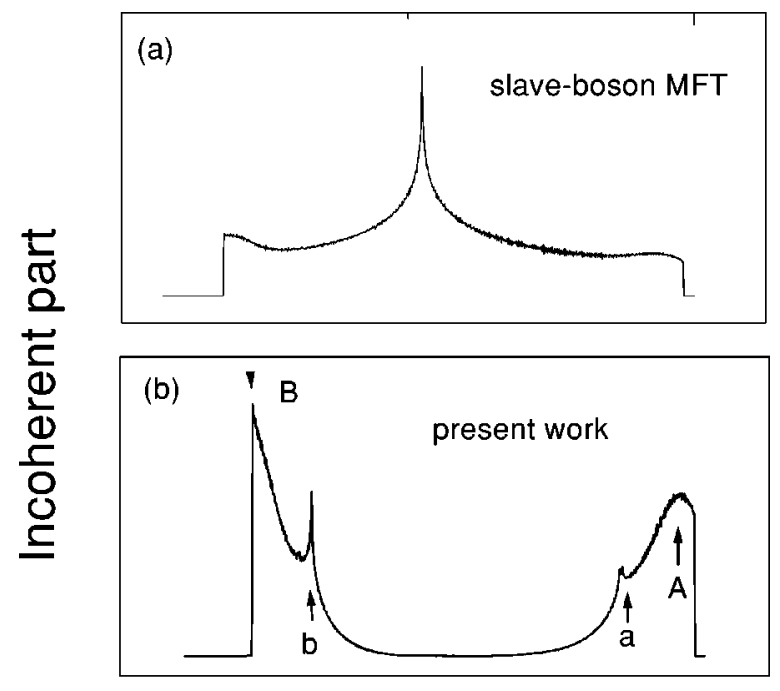

$\omega$ (arb. units)

FIG. 4. Incoherent part of the spectral function at $\mathbf{k}_{f}=(0.4, \pi)$ : (a) calculation based on the slave-boson $d$-wave RVB mean-field state; (b) the present theory. Note the additional structure in (b). All energies have been scaled by $1 \mathrm{eV}$, where peak $B$ occurs in our calculations for our choice of parameters.

along the direction $\Gamma \rightarrow(\pi, \pi)$ are remarkably similar to the results obtained from ARPES of optimally doped $(x=0.15)$ $\mathrm{La}_{2-x} \mathrm{Sr}_{x} \mathrm{CuO}_{4} \cdot{ }^{16}$ Finally, we remark that the effects of doping within our calculation enter only through the choice of our parameters, $t_{h}$ and the chemical potential $\mu$. So, the positions of the peak and hump would change continuously with doping, but no qualitative changes are expected. However, for low enough doping, the mean-field theory loses its validity. In this regime, the ground state is characterized by inhomogeneous charge distribution and our results do not hold.

In Fig. 4, we show the results for the incoherent part $A_{-}^{\mathrm{i}}(\mathbf{k}, \omega)$ at $\mathbf{k}_{f}=(0.4, \pi)$ near the $M$ point. To highlight the importance of the short-range AF correlations, we show results obtained with the inclusion of the prefactor $\eta_{\mathbf{k}}$ (our calculation) and without this prefactor (analogous to the slave-boson theories). We choose the same set of parameters in obtaining the results. However, to enable direct comparison between the two results, we have scaled the energies by the value of energy for which our calculation exhibits a highenergy peak $(1 \mathrm{eV})$. Figure 4(a) shows the results without the inclusion of the AF correlations, and Fig. 4(b) shows our results. The differences can be seen over a wide range of energies. Besides the hump structure at low energy [indicated by the letter $A$ in Fig. 4(b)], there is also another hump $(B)$ at the high-energy region of the spectrum (which is approximately $1 \mathrm{eV}$ for our choice of parameters, as mentioned earlier). In contrast, Fig. 4(a) shows a broad incoherent structure that is almost featureless over the same energy range. Clearly, the static prefactor $\eta_{\mathbf{k}}$ in the spinon propagator, resulting from the local AF spin oscillations around the hole, strongly modifies the lineshape (hence the dynamics) of the 
electron spectral function, since the latter is a convolution of the holon and spinon spectral functions. Finally, we draw the readers' attention to the two satellite peaks ( $a$ and $b$ ) in Fig. 4(b). These peaks arise because the holons see a $\pi$ flux per plaquette according to Eq. (4). We expect these peaks to be smeared out when interactions between the holons are taken into account.

To conclude, we presented a calculation of the spectral function in a superconducting RVB state. The calculation is based on the phase-string mean-field theory of the $t$ - $J$ model. The key observation of this paper is that the presence of a hole in an RVB environment induces short-range AF oscillations. We incorporated this feature within our calculation and showed that it leads to strong matrix element effects in the hole channel. In particular, we showed that the spectral function shows the peak-dip-hump structure near the $M$ point of the Brillouin zone. This structure gets progressively weaker, owing to the aforesaid matrix element effect, as we move in the direction $\Gamma \rightarrow(\pi, \pi)$. Our calculation also illustrates that the peak-dip-hump structure seen in the insulating cuprate has the same origin as in the optimally doped cuprates. In the former, the matrix element effects described in this paper reduce to the "coherence factors" effect of the Schwinger Boson mean-field theory of the Heisenberg model. ${ }^{12}$ Thus, our scheme provides a natural interpolation between the description of the undoped and the optimally doped cuprates. In a forthcoming paper, we shall extend the results presented in this paper to describe the AF correlations induced by the addition of $\mathrm{Zn}$ impurities to the cuprate superconductors.

\section{ACKNOWLEDGMENTS}

We thank P. W. Anderson for his comments. We are also thankful to H. Ding, A. Fedorov, P. D. Johnson, T. Valla and B. O. Wells for several discussions on ARPES. V.N.M. is supported by NSF Grant No. DMR-9104873. Z.Y.W. is supported by NSFC Grant No. 90103021, and also acknowledges partial support from TCSUH, where part of this work was carried out.

${ }^{1}$ For a recent review, see, J.C. Campuzano, More is Different, Princeton Series in Physics (Princeton University Press, Princeton, 2001).

${ }^{2}$ A.V. Fedorov, T. Valla, P.D. Johnson, Q. Li, G.D. Gu, and N. Koshizuka, Phys. Rev. Lett. 82, 2179 (1999).

${ }^{3}$ D.L. Feng, D.H. Lu, K.M. Shen, C. Kim, H. Eisaki, A. Damascelli, R. Yoshizaki, J.-i. Shimoyama, K. Kishio, G.D. Gu, S. Oh, A. Andrus, J. O'Donnell, J.N. Eckstein, and Z.-X. Shen, Science 289, 277 (2000).

${ }^{4}$ H. Ding, J.R. Engelbrecht, Z. Wang, J.C. Campuzano, S.C. Wang, H.-B. Yang, R. Rogan, T. Takahashi, K. Kadawoki, and D.G. Hinks, cond-mat/0006143 (unpublished).

${ }^{5}$ T. Valla, A.V. Fedorov, P.D. Johnson, B.O. Wells, S.L. Hubert, Q. Li, G.D. Gu, and N. Koshizuka, Science 285, 2110 (1999).

${ }^{6}$ Takami Tohyama and Sadamichi Maekawa, Supercond. Sci. Technol. 13, R17 (2000).

${ }^{7}$ P.W. Anderson, The Theory of Superconductivity in the High $T_{c}$ Cuprates (Princeton University Press, Princeton, 1997).

${ }^{8}$ Z.Y. Weng, V.N. Muthukumar, D.N. Sheng, and C.S. Ting, Phys. Rev. B 63, 075102 (2001).

${ }^{9}$ Z.Y. Weng, D.N. Sheng, and C.S. Ting, Phys. Rev. Lett. 80, 5401 (1998); Phys. Rev. B 59, 8943 (1999).

${ }^{10}$ Z.Y. Weng, D.N. Sheng, and C.S. Ting, Phys. Rev. B 61, 12328 (2000).

${ }^{11}$ P.A. Lee and N. Nagaosa, Phys. Rev. B 46, 5621 (1992); X.G. Wen and P.A. Lee, Phys. Rev. Lett. 76, 503 (1996); Jian-Xin Li, Chung-Yu Mou, and T.K. Lee, Phys. Rev. B 62, 640 (2000); C. Lannert, M.P.A. Fisher, and T. Senthil, cond-mat/0101249 (unpublished).

${ }^{12}$ D.P. Arovas and A. Auerbach, Phys. Rev. B 38, 316 (1988); Daijiro Yoshioka, J. Phys. Soc. Jpn. 58, 32 (1989).

${ }^{13}$ T. Valla (unpublished).

${ }^{14}$ Hung Fai Fong, B. Keimer, P.W. Anderson, D. Reznik, F. Dogan, and I.A. Aksay, Phys. Rev. Lett. 75, 316 (1995).

${ }^{15}$ J.C. Campuzano, Phys. Rev. Lett. 83, 3709 (1999).

${ }^{16}$ Akihiro Ino, Changyoung Kim, Takashi Mizokawa, Zhi-Xun Shen, Atsushi Fujimori, Masamitsu Takaba, Kenji Tamasaku, Jiroshi Eisaki, and Shinichi Uchida, J. Phys. Soc. Jpn. 68, 1496 (1999). 